\title{
Type-2 Fuzzy Logic Controller Based PSS for Large Scale Power Systems Stability
}

\author{
O. Kahouli \\ Electrical Engineering \\ Department, National \\ Engineering School of \\ Sfax, University of Sfax, \\ Sfax, Tunisia \\ omarkahouli@yahoo.fr
}

\section{B. Ashammari}

Electrical Engineering

Department, College of Engineering,

University of Hail,

Hail, Saudi Arabia

badr_ms@hotmail.com

\author{
K. Sebaa \\ Electrical and Computer \\ Sciences Engineering \\ Department, \\ University of Medea, \\ Medea, Algeria
}

\author{
M. Jebali \\ Electrical Engineering \\ Department, National \\ Engineering School of \\ Sfax, University of Sfax, \\ Sfax, Tunisia

\begin{abstract}
H. Hadj Abdallah
Electrical Engineering

Department, National

Engineering School of Sfax, Tunisia hsan.haj@enis.rnu.tn
\end{abstract} \\ Sfax, University of Sfax,
}

\begin{abstract}
In this paper, the application of the fuzzy logic based power systems stabilizer (FLPSS) to damp power system oscillation is presented. Various types of fuzzy logic controller are used to replace the conventional power system stabilizer (CPSS). The classic fuzzy logic controller based PSS (FLCPSS), the polar FLC (PFLCPSS) and the interval type-2 fuzzy logic controller based PSS (IT2FLCPSS) are applied to the New England - New York interconnected power system and the obtained results are compared. For coordination purposes, genetic algorithm (GA) is used to tune the FLCPSS's gains. The non-linear simulation in the presence of noise confirms the robustness and the superiority of the IT2FLCPSS.
\end{abstract}

Keywords-CPSS; FLCPSS; PFLCPS; IT2FLPSS; GA; prony analysis

\section{INTRODUCTION}

Nowadays, power systems are driven to work around their limits of stability in a fast and flexible way. Hence, modern power systems may reach disagreeable conditions like poorly damped electromechanical oscillations. A possible change in the operating point could lead to weak interconnected lines. These lines will cause the generation of inter-area oscillations with poorly damped low frequency modes. Improving the damping of these modes by the power systems stabilizers (PSSs) is an open research topic. PSSs are designed around some operating points, with the change in these operating points leading to un-damped oscillations, or even to instability. Recently, fuzzy logic controllers (FLCs) have attracted considerable attention as candidates for novel control strategies because of their advantages over conventional computational systems. Unlike other classical control methods, FLCs are model-free controllers, i.e. they do not require an exact mathematical model of the controlled system. Moreover, rapidity and robustness are their most profound and interesting properties in comparison to the classical schemes. Designing PSSs based on FLC has become an active area and satisfactory results have been obtained [1-3]. These FLCPSSs use the concept of the T1FLS (type-1 fuzzy logic system). The concept of the T2FLS (type 2 fuzzy logic system) was initially proposed as an extension of the classical T1FLS. T2FLS is very useful in situations where it is difficult to determine an exact membership function (MF) for a fuzzy set. Hence, it is very effective for dealing with uncertainties, such as those due to penetration of the distributed generation in power systems. However, T2FLSs are more difficult to use and understand than T1FLS. Despite these difficulties, T2FLS has found applications in many fields [4-9]. The concept of the IT2FLCPSS (interval type-2 fuzzy logic controller PSS) has been introduced in power systems during the last decade. Authors in [10] presented the use of the T2FS based adaptive synergetic PSS to improve the stability of the power system. Others proposed a PSS based on T2FLS to improve power system stability where differential evolution algorithm is applied to optimize the IT2FLCPSS parameters and rule base $[11,12]$. On the other hand, for improvement of the voltage stability, an IT2FLC is proposed and tuned based on the approximation of the system by the T1 TS fuzzy model [13]. Its drawback is that the application of the IT2FLPSS is only suitable for small scale power systems in which authors approximated the dynamics of the power system by the T1TSK (type 1 Takagi, Sugeno, and Kang) fuzzy model which is then used to tune the IT2 FLC via the LMI approach. This method is tested on small systems (two machines) with the third-order single-axis dynamic model. For realistic large scale power systems with power electronic devices this method is time consuming and not easy to be applied as it requires:

1. Two independent highly nonlinear functions by generator for building of the T1TSF approximation, which is difficult to obtain for large scale power systems with various power electronic devices,

2. Four state feedback gains and two controller parameters tuning for each generator. Also their work is related to the voltage control.

In [14] an interval type-2 fuzzy controller-based thyristor controlled series capacitor (IT2TCSC) has been used alongside CPSS for improving power system stability on large scale power systems to damp out the speed and power oscillations 
following different critical faults. They use: i) The active power line as an input of the IT2TCSC as opposed to the generator speed, for study of the power system oscillation. ii) The IT2TCSC use of the sliding surface that leads to the charting problem. iii) Implementation of two IT2TCSC with the presence of CPSS.

Based on the research described above, our goal is the damping of the power system's oscillation. The proposed method requires only three gains per generator. Then, attempts will be made to demonstrate that it can be applied to realistic power systems, and used for solving the coordination of IT2FLCPSS for large scale power systems. Hence, various types of PSS controllers namely FLCPSS [2], PFLCPSS [1], CPSS and our contribution of IT2FLCPSS are presented in this paper. This is achieved by applying the previously mentioned FLCPSSs to a 68-bus, 16-machine power system, which is large and close to realistic power systems. Several scenarios are considered to verify the robustness of the proposed method. The nonlinear simulation and eigenvalue analysis demonstrate the significant improvement of dynamic oscillations by the IT2FLCPSS under each scenario.

\section{TYPE-2 FUZZY LOGIC SYSTEM DESIGN}

The structure of the T2FLS is shown in Figure 1. T2FLS is similar to T1FLS. The main difference between them is that T2FLS requires a type-reduced process to convert the output of the fuzzy inference engine into a type-reduced set and at least one of the fuzzy sets is T2. The crisp output is obtained by the defuzzyfication of the type-reduced set. The T2FLS can efficiently simplify the computational process of type-reduction and is very simple to use [5].

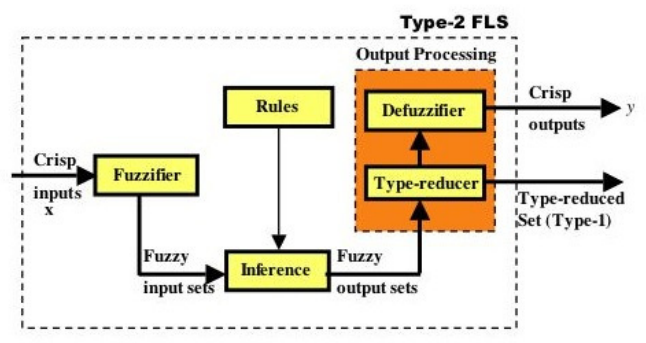

Fig. 1. Structure of a T2FLS

Consider a type-2 TSK fuzzy logic system having $\mathrm{n}$ inputs, $x=\left[x_{1}, \ldots, x_{n}\right] \in X_{1} \times \ldots \times X_{n}$ and one output $y \in Y$. In order to construct the fuzzy rules we assume that there are $\mathrm{M}$ rules in the type- 2 fuzzy system, where the $i^{\text {th }}$ rule has the following form:

$$
R^{i}: \text { if } x_{1} \text { is } \tilde{F}_{1}^{i} \text { and } \ldots \text { and } x_{n} \text { is } \tilde{F}_{n}^{i} \text { Then } y^{i}=C^{i}
$$

where $\tilde{F}_{1}^{i}, \tilde{F}_{2}^{i}, \ldots, \tilde{F}_{n}^{i}$ are antecedent linguistic terms modelled by the interval type- 2 triangular fuzzy sets (Figure 2), $y^{i}$ is the output of $i^{\text {th }}$ rule $R^{i}$ and the consequent parameter $C^{i}$ is an interval type- 1 set. In Figure 2, the footprint of uncertainty (FOU) of each membership function (MF) can be represented as a bounded interval in terms of the upper MF $\bar{\mu}_{\tilde{F}_{j}^{i}}\left(x_{j}\right)$ and the lower MF $\underline{\mu}_{\tilde{F}_{j}^{i}}\left(x_{j}\right)$, where:

$$
\begin{aligned}
& \bar{\mu}_{\tilde{F}_{j}^{i}}\left(x_{j}\right)=\max \left(\min \left(\frac{x_{j}-a_{j}}{b_{j}-a_{j}}, \frac{c_{j}-x_{j}}{c_{j}-b_{j}}\right), 0\right) \\
& \equiv \operatorname{tri}\left(x_{j}, \mathrm{a}_{j}, \mathrm{~b}_{j}, \mathrm{c}_{j}\right) \\
& \text { and } \underline{\mu}_{\tilde{F}_{j}^{i}}\left(x_{j}\right)=0.8 \cdot \bar{\mu}_{\tilde{F}_{j}^{i}}\left(x_{j}\right)
\end{aligned}
$$

$a_{j}, b_{j}$ and $c_{j}$ are the parameters of triangular primary MF of the type-2 fuzzy set $\tilde{F}_{j}^{i}$.

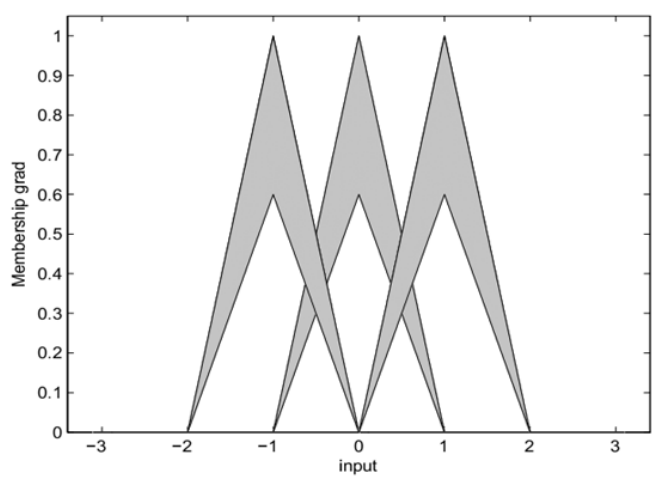

Fig. 2. Interval type-2 triangular MFs for antecedents sets.

The inference engine combines the fuzzy rules in order to map the crisp inputs to interval type- 2 fuzzy output sets. Based on the input and the antecedents of the rules, it calculates a firing interval for each rule and then applies these firing levels to the consequent fuzzy sets. The firing interval $\left[\underline{f}^{i}, \bar{f}^{i}\right]$ of the $i^{\text {th }}$ rule is an interval type- 1 set, which is determined by its left-most and right-most points $\underline{f}^{i}$ and $\bar{f}^{i}$ such that :

$$
\begin{aligned}
& \underline{f}^{i}=\underline{\mu}_{\tilde{F}_{1}^{i}}\left(x_{1}\right) * \underline{\mu}_{\tilde{F}_{2}^{i}}\left(x_{2}\right) * \ldots * \underline{\mu}_{\tilde{F}_{n}^{i}}\left(x_{n}\right) \\
& \bar{f}^{i}=\bar{\mu}_{\tilde{F}_{1}^{i}}\left(x_{1}\right) * \bar{\mu}_{\tilde{F}_{2}^{i}}\left(x_{2}\right) * \ldots * \bar{\mu}_{\tilde{F}_{n}^{i}}\left(x_{n}\right)
\end{aligned}
$$

where $\underline{\mu}_{\tilde{F}_{j}^{i}}\left(x_{j}\right)$ and $\bar{\mu}_{\tilde{F}_{j}^{i}}\left(x_{j}\right)$ represent the membership values of the lower and the upper membership functions of the crisp input $x_{j}$ to the type- 2 fuzzy set $\tilde{F}_{j}^{i}$ in the $i^{\text {th }}$ rule. When interval type-2 fuzzy sets are used for the antecedents, and interval type-1 fuzzy sets are used for the consequent sets of Type-2 TSK rules, the final output can be expressed as $[4,5]$ :

$$
Y=\left[y_{l}, y_{r}\right]
$$

The output $Y$ is an interval type- 1 set, therefore, only its two endpoints $y_{l}$ and $y_{r}$ need to be computed which can be 
represented as an expansion of fuzzy basis functions (FBFs), as:

$$
\begin{aligned}
& y u_{l}=\sum_{i=1}^{M} f_{l}^{i} \cdot u_{l}^{i} / \sum_{i=1}^{M} f_{l}^{i} \\
& y_{r}=\sum_{i=1}^{M} f_{r}^{i} \cdot u_{r}^{i} / \sum_{i=1}^{M} f_{r}^{i}
\end{aligned}
$$

where $f_{l}^{i}$ and $f_{r}^{i}$ denote the firing strength membership grades contributing to the left-most-point $y_{l}$ and the right-most-point $y_{r}$ respectively, $u_{l}^{i}$ and $u_{r}^{i}$ are the singleton lower and upper control actions of the consequences part. From the typereduction stage a type-reduced set exists for each output. The crisp output of the controller is equal to the mind point of the type-reduce set:

$$
y_{c}=\frac{y_{l}+y_{r}}{2}
$$

using the iterative procedure given in [15]. The computation procedure is briefly provided below.

Initially, the right-most point $y_{r}$ is computed. Without loss of generality, it is assumed that the parameters $u_{i}$ are arranged in ascending order, i.e., $u_{1} \leq u_{2} \leq u_{3} \ldots \ldots \leq u_{M}$

- Step 1: Compute $y_{r}$ in (7) by initially setting $f_{r}^{i}=\left(\underline{f}^{i}+\bar{f}^{i}\right) / 2$, for $i=1,2, \ldots \ldots ., M$ and let $y_{r}^{\prime}=y_{r}$

- Step 2: Find $k \quad(1 \leq k \leq M-1)$ such that $u^{k} \leq y_{r}^{\prime} \leq u^{k+1}$.

- Step 3: Compute $y_{r}$ in (7) with $f_{r}^{i}=\underline{f}^{i}$ for $i \leq k$ and $f_{r}^{i}=\bar{f}^{i}$ for $i>k$, then set $y_{r}^{\prime \prime}=y_{r}$.

- Step 4: If $y_{r}^{\prime \prime} \neq y_{r}^{\prime}$, then go to step 5. If $y_{r}^{\prime \prime}=y_{r}{ }^{\prime}$ then set $y_{r}=y_{r}^{\prime \prime}$ and go to step 6 .

- Step 5: Set $y_{r}^{\prime}=y_{r}^{\prime \prime}$ and return to step 2.

- Step 6: End.

The procedure to compute $y_{l}$ is very similar, only two changes need to be made. In step 2 , we need to find $1 \leq k^{\prime} \leq M-1$ such that $u^{k} \leq y_{l}^{\prime} \leq u^{k+1}$, and in step 3, let $f_{l}^{i}=\underline{f}^{i}$ for $i \leq k^{\prime}$ and $f_{l}^{i}=\bar{f}^{i}$ for $i>k^{\prime}$.

\section{FUZZY LOGIC BASED PSS}

Selection of the input variables of the FLC based PSS depends on the nature of the controlled plant and the desired outputs. Generally, it is common to use the output error and their derivative (or their integral). Since the goal of this work is the improvement of the system damping, the speed deviations and their derivative are used as shown in Figure 3.

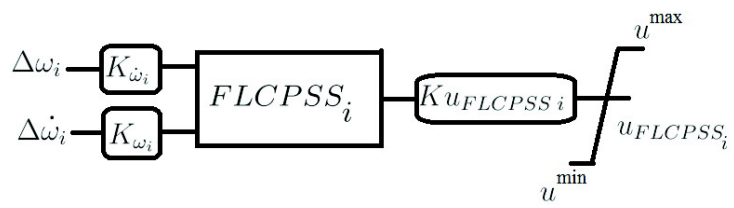

Fig. 3. Fuzzy logic based PSS.

\section{A. FLCPSS and IT2FLC}

The structure of these two controllers is similar, with small differences. The speed deviation $\Delta \omega_{i}$ and acceleration deviation $\Delta \dot{\omega}_{i}$ of $i^{\text {th }}$ generator are used as the inputs of the FLCPSS and the IT2FLCPSS in this paper. The output control signal from the FLCPSS ( $U_{F L C P S S_{i}}$ ) is injected to the summing point of the automatic voltage regulator (AVR) where an eventual CPSS should be connected. To convert the measured input variables of the FLCPSS into suitable linguistic variables, seven fuzzy subsets NB, NM, NS, ZO, PS, $\mathrm{PM}, \mathrm{PB}$ are chosen. The MFs for the input variables, as used in the present study, are shown in Figure 4. In this study, both inputs of FLCPSS and IT2FLCPSS have seven subsets. Thus, a fuzzy table consisting of forty nine rules should be constructed. Table I shows a rule table obtained by the trial

\begin{tabular}{|c|c|c|c|c|c|c|c|c|}
\hline & \multicolumn{7}{|c|}{$\Delta \omega$} \\
\hline & & NB & NM & NS & $\mathrm{ZO}$ & PS & PM & PB \\
\hline \multirow{7}{*}{$\Delta \dot{\omega}$} & NB & $\mathrm{NL}$ & $\mathrm{NL}$ & $\mathrm{NL}$ & NM & $\mathrm{MN}$ & NS & $\mathrm{ZO}$ \\
\hline & NM & $\mathrm{NL}$ & $\mathrm{NL}$ & $\mathrm{NM}$ & NM & NS & $\mathrm{ZO}$ & PS \\
\hline & NS & $\mathrm{NL}$ & $\mathrm{NM}$ & NS & NS & $\mathrm{ZO}$ & PS & $\mathrm{PM}$ \\
\hline & $\mathrm{ZO}$ & NM & NM & NS & $\mathrm{ZO}$ & PS & PM & PM \\
\hline & PS & NM & NS & $\mathrm{ZO}$ & PS & PS & PM & PL \\
\hline & PM & NS & $\mathrm{ZO}$ & PS & PM & PM & PL & PL \\
\hline & PB & $\mathrm{ZO}$ & PS & PM & PM & $\mathrm{PL}$ & PL & $\mathrm{PL}$ \\
\hline
\end{tabular}
error based on the result obtained from the CPSS.

TABLE I. RULES FOR FLCPSS AND IT2FLCPSS

The triangular shape function is chosen to follow the work in $[1,2]$. The Gaussian shape function can also be used. However in our study, for better performance, triangular shape and $7 \mathrm{MFs}$ are used. Quicker response is observed with $3 \mathrm{MFs}$, but with poorer performance. The best compromise between performance and simulation time is realized by $7 \mathrm{MFs}$ as is also suggested in [2]. In order to take a crisp control action, the fuzzy control action inferred from the fuzzy control algorithm must be defuzzyfied. To ensure that all the fired rules have some contribution in the control action, the method of the center of gravity is employed in this study. IT2FLCPSS requires a reduction strategy. The reduction used in this paper is centroid method. The input gains $K \omega_{i}, K \dot{\omega}_{i}$ and the output gain $K u_{\mathrm{FLCPSS}_{i}}$, are used to properly scale the fuzzy input and output variables. 


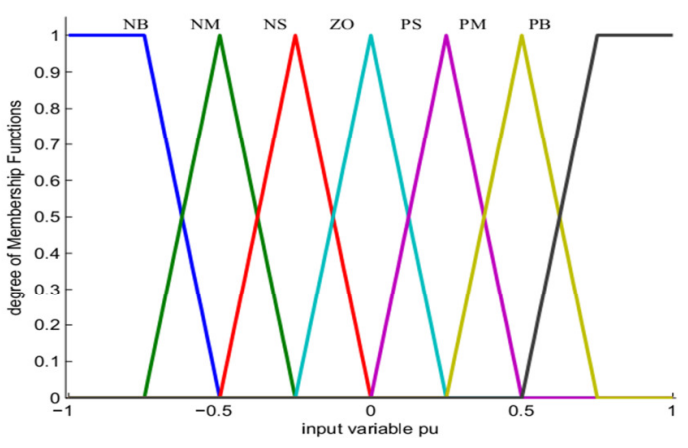

(a) Membership functions for FLCPSS

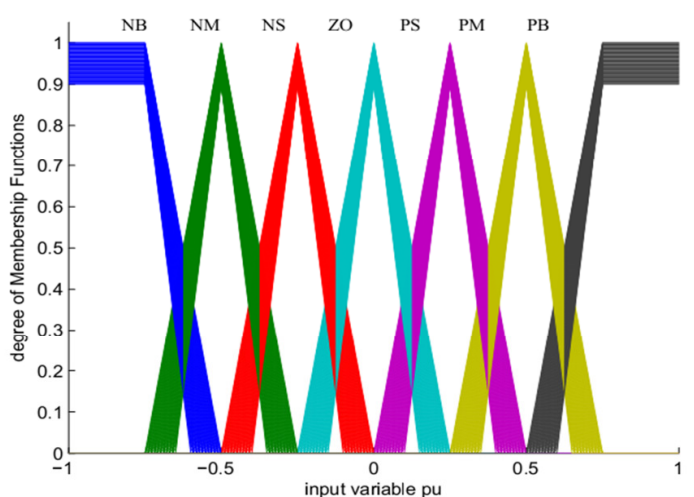

(b) Membership functions for IT2FLCPSS

Fig. 4. Membership functions.

\section{B. PFLCPSS}

PFLCPSS has been originally developed in [1]. The concept of this controller is based on the sign of $\Delta \omega$ and $\Delta \dot{\omega}$. Two membership functions are created $(N(\theta), P(\theta))$ to represent four situations. The stabilizing signal is calculated using the following steps:

1. Determine the piecewise linear fuzzy MFs $N(\theta)$ and $P(\theta)$ as described in [1] and reproduced in Figure 5. These MFs use the angle $\theta$ obtained from the phase plane $\left(\theta=\tan ^{-1}(\Delta \dot{\omega} / \Delta \omega)\right)$.

2. Compute the stabilizing signal $U(k)$ using

$$
U(k)=G c(k) \cdot \frac{N(\theta)-P(\theta)}{N(\theta)+P(\theta)} \cdot U_{\max }
$$

where $G c(k)$ is the gain whose value is given by (10):

$$
G c(k)=\left\{\begin{array}{cc}
R=\sqrt{\Delta \omega^{2}+\Delta \dot{\omega}^{2}} & \text { if } R<D r \\
1 & \text { otherwise }
\end{array}\right.
$$

Parameter $D_{r}$ should be adjusted to it optimal value. For optimal parameter setting, various performance indices can be used and minimized by traditional or meta-heuristic methods. In this paper, $D_{r}$ is equal to 0.98 . The value of Umax depends on the synchronous machine to be studied (it is equal to $0.02 \mathrm{pu}$ in this study). We call this stabilizer Polar FLPCSS.

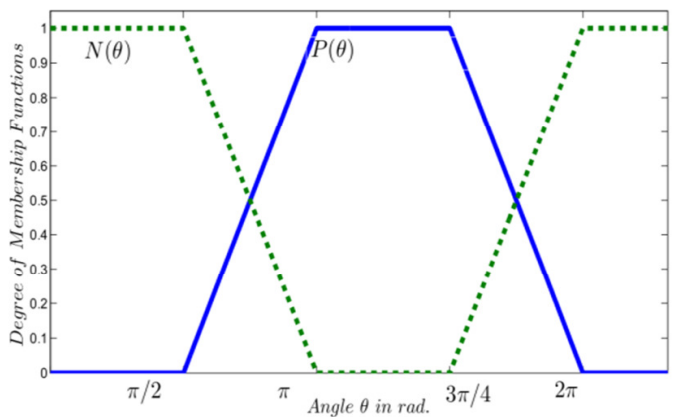

Fig. 5. Membership functions for PFLCPSS

\section{CPSS}

The PSS or CPSS is employed to add supplementary damping to the rotor oscillations of the synchronous machine by controlling its excitation. The electromechanical oscillations of the electrical generators must be effectively damped to maintain the system stability. The output signal of the PSS is introduced as an additional signal to the AVR $\left(U_{P S S}\right)$ to the excitation system block. The PSS input signal can be the machine speed deviation. The PSS can be modeled by the following non-linear system (Figure 6):

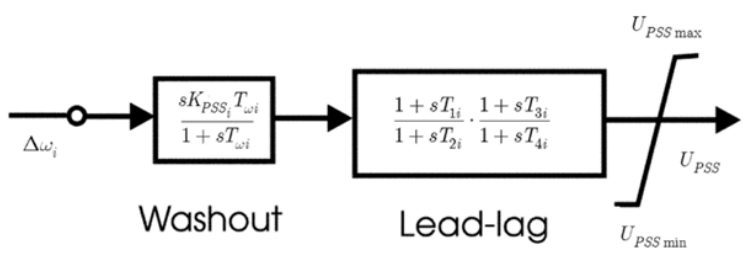

Fig. 6. Conventional power system stabilizer model block diagram.

To obtain an adequate damping, PSS should provide a moderate phase advance at frequencies of interest in order to compensate for the inherent lag between the field excitation and the electrical torque induced by the PSS action. The model consists of a low-pass filter, a general gain, a washout highpass filter, a phase-compensation system, and an output limiter. The gain KPSS determines the amount of damping produced by the stabilizer. The washout filter eliminates low frequencies that are present in the $\Delta \omega$ signal and allows the PSS to respond only to speed changes. The phase-compensation system is represented by a cascade of two first-order lead-lag transfer functions used to compensate the phase lag between the excitation voltage and the electrical torque of the synchronous machine. For comparison, a $\Delta \omega$ conventional type PSS (CPSS) is designed under the same scenarios used in [16]. CPSSs parameters can be obtained by the classical method of searching for the close fit to the ideal case from a number of phase compensator characteristics. PSS parameters are computed by the root locus method [17]. However, in this paper PSS parameters are taken from [16], in which GA was used to find the parameters that give the best damping ratio $\xi$. 


\section{MULTIMACHINE POWER SYSTEM MODEL}

A 16-machine power system, as shown in Figure 7, is used to test the proposed IT2FLCPSS. This power system comprises of five coherent areas representing a reduced model of the New England and New York interconnected system. The solid lines indicate the major weak tie lines that cause the low frequency inter-area oscillations. Each of the areas 1,2 and 3 contains a single large generator, which is represented by its aggregated equivalent model. Area 4 is modeled with an equivalent generator in addition to more normally sized generators. Area 5 has the most detailed modeling that includes static exciters, thermal turbines and governors on all generators. The power system is modeled by a set of non-linear differential equations [18]:

$$
\dot{x}=f(x, u)
$$

where $x$ is a vector state $x=\left[\delta, \omega, E_{q}^{\prime}, \psi_{d}^{\prime \prime}, E_{d}^{\prime}, \psi_{s}^{\prime \prime}\right]$ and $u$ is the vector of the PSS, IT2FLCPSS, PFLCPSS or FLCPSS output signals. Details of network parameters are given in [17]. To assess the FLCPSSs four scenarios are tested (Table II).

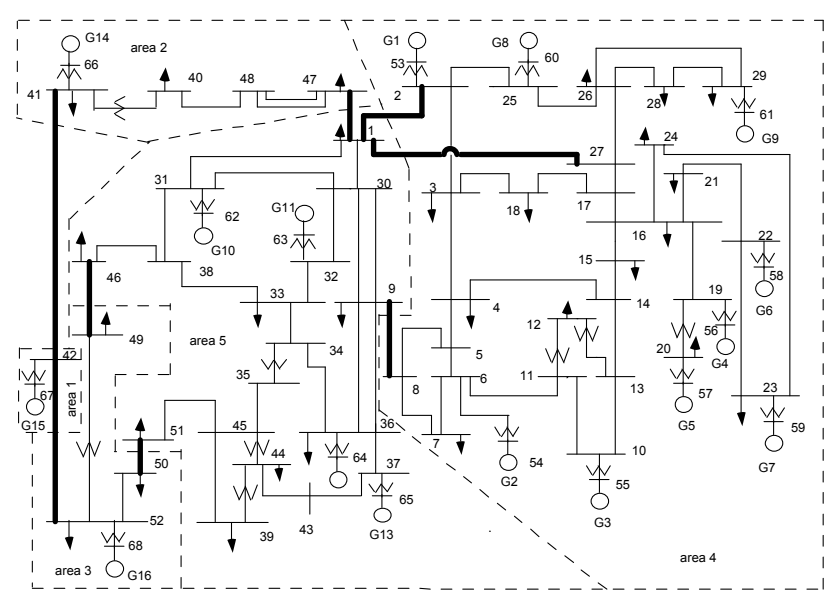

Fig. 7. New England - New York interconnected power system model

TABLE II. SCENARIOS CONSIDERED

\begin{tabular}{|c|c|}
\hline Scenario no & Description \\
\hline 1 & Line 28-29 out of service \\
\hline 2 & Line $1-2$ out of service \\
\hline 3 & Lines $25-26$ and $3-18$ out of service \\
\hline 4 & Line $41-42$ out of service \\
\hline
\end{tabular}

\section{A. Tuning of the Gains}

Each fuzzy logic controller requires an adequate tuning of the input gains $K_{\omega_{i}}, K_{\dot{\omega}_{i}}$ and the output gains $K u_{F L C P S S} i$ (Figure 3). These gains are used to properly scale the fuzzy input and output variables of the FLC based PSS. Generally, these gains can be obtained by a tedious trial-and-error process for small size systems. But for large scale systems the use of such a technique is impossible. As the aim of this work concerns large scale power systems, an optimization based GA method is proposed to compute these parameters. The objective of the off-line tuning algorithm is to change the controller gains to obtain the desired system response. The tuning algorithm tries to minimize the system overshoot index $\mathrm{J}$ :

$$
\min J\left(K \omega_{i}, K \dot{\omega}_{i}, K u_{F L C P S S_{i}}\right)=\sum_{k=1}^{T / T s} \sum_{i=1}^{16} \Delta \omega_{i}(k)
$$

where $T$ is the simulation time (11 seconds). It is very time consuming to use the non-linear simulation. For this reason the linearized power system is used to speed-up this process. The main elements of the GA used to resolve this optimization problem are defined in [16]. GA is stopped when a maximum number of generations $(100)$ is reached. Obtained parameters by the GA are summarized in Table III. From Figure 8 it is clear that this solution is stable as it is reached at 60 generations. Table III shows the input and the output gains. Without control, the eigenvalue analysis confirms that the power system is unstable $[16,19]$. Four contingencies are applied to assess the performance of the four controllers (CPSS, FLCPSS, PFLCPSS and IT2FLCPSS). The parameters of CPSS for generators 1 to 16 are obtained using the GA procedure [16].

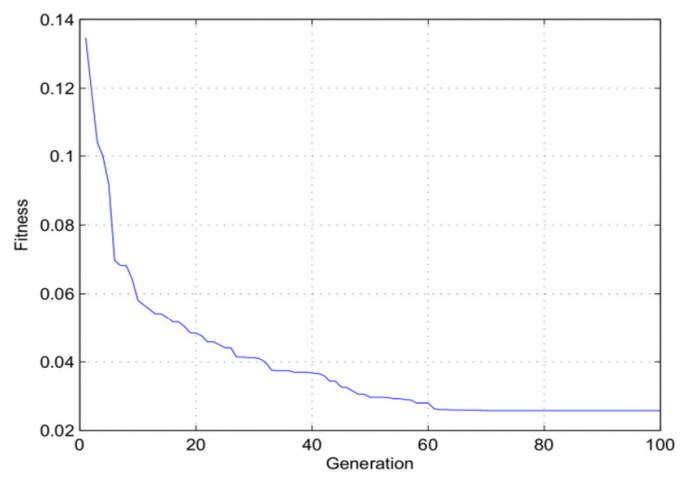

Fig. 8. Convergence of the GA optimization

\begin{tabular}{|c|c|c|c|c|c|c|c|}
\hline mach. & $K_{\omega_{\iota}}$ & $\boldsymbol{K}_{\dot{\omega}_{i}}$ & $K u_{F L C i}$ & mach. & $K_{\omega_{i}}$ & $K_{\dot{\omega}_{i}}$ & $K u_{F L C i}$ \\
\hline \#01 & 4.26 & 2.51 & 0.95 & \#09 & 2.86 & 8.36 & 0.98 \\
\hline \#02 & 3.42 & 6.62 & 0.95 & $\# 10$ & 1.38 & 4.82 & 0.81 \\
\hline \#03 & 4.05 & 4.17 & 0.93 & \#11 & 1.46 & 3.02 & 0.87 \\
\hline \#04 & 3.28 & 4.26 & 0.78 & $\# 12$ & 3.12 & 3.04 & 0.89 \\
\hline$\# 05$ & 2.04 & 6.97 & 0.85 & $\# 13$ & 4.45 & 1.35 & 0.83 \\
\hline$\# 06$ & 1.24 & 4.57 & 0.99 & $\# 14$ & 2.79 & 0.39 & 0.66 \\
\hline$\# 07$ & 3.72 & 6.12 & 0.96 & $\# 15$ & 2.15 & 1.81 & 0.41 \\
\hline$\# 08$ & 0.39 & 6.28 & 0.79 & $\# 16$ & 3.34 & 2.82 & 0.31 \\
\hline
\end{tabular}

TABLE III. GAINS CONSTANTS

The 1st (resp. 2nd, 3rd) contingency consists on the application of the three-phase to ground fault in line 1-2 (resp. line 28-29, line 41-42) at 0.1s, and cleared after 6 cycles. In the fourth contingency, the application of the three-phase to ground fault in line $3-18$ at $0.1 \mathrm{~s}$ is considered, which is then cleared after 6 cycles. For the system with CPSS, linear analysis is carried out by the perturbation method for building the state space system, after which the modes correspond to the eigenvalues of the linearized system. However, in the presence of IT2FLCPSS the modes are obtained by Prony analysis as it is not possible to use the perturbation method, as in the case of 
the presence of CPSS. Prony analysis is a method for extracting sinusoidal exponential signals from time series data, by solving a set of linear equations. Assuming $\mathrm{N}$ complex data samples $X[1], \ldots, X[N]$ the investigated function can be fitted by $M$ exponential functions:

$$
\hat{f}(t)=\sum_{i=1}^{N} \frac{1}{2} A_{i} e^{\lambda_{i}^{t j} \phi_{i}}
$$

In order to perform the damping of the power system oscillations by PSSs, several sensors are necessary. However, sensor readings are often noisy. In order to simulate the impact of the measurement uncertainty on the performance of T1 FLC based PSS and T2 FLC based PSS, random noise with normal distribution is added to the measurement values of rotating speed and acceleration. To carry out a quantitative comparison between the controllers, three well known performance criteria are used [5]: the integral of square error, ISE $=\sum_{n=1}^{16} \int \Delta \omega_{n}^{2}(t) d t$, the integral of the absolute value of the error, IAE $=\sum_{n=1}^{16} \int\left|\Delta \omega_{n}(t)\right| d t$, and the integral of the time multiplied by the absolute value of the error, ITAE $=\sum_{n=1}^{16} \int t \cdot\left|\Delta \omega_{n}(t)\right| d t$. Generally, these indexes of performance are used in the optimal tuning of the controller. In this work, these will be employed to assess the stability of the closed loop system. Minimum value of ISE means that the controller will eliminate large errors quickly, but will tolerate small errors persisting for a long period. A system with minimum IAE tends to produce slower response than ISE optimal systems, but usually with less oscillation. A system with small ITAE settles much more quickly than the other two errors. In Table IV, the values for ISE, IAE and ITAE are summarized considering noise of $0.02 \mathrm{pu}$ and $0.05 \mathrm{pu}$ applied to the speeds and accelerations of all 16 machines and only in the case of the first contingency.

TABLE IV. PERFORMANCE CRITERIA VALUES UNDER 1ST CONTINGENCY IN PRESENCE OF 0.02PU AND 0.05PU NOISE

\begin{tabular}{|c|c|c|c|c|c|}
\hline \multicolumn{2}{|c|}{} & \multicolumn{2}{c|}{ Noise of 0.02pu } & \multicolumn{2}{c|}{ Noise of 0.05pu } \\
\cline { 3 - 6 } \multicolumn{2}{|c|}{} & Avg. Value & Std. Dev. & Avg. Value & Std. Dev. \\
\hline \multirow{3}{*}{ CPSS } & ISE & $2.46 \mathrm{e}-02$ & $5.60 \mathrm{e}-03$ & $3.49 \mathrm{e}-02$ & $3.55 \mathrm{e}-02$ \\
\cline { 2 - 6 } & IAE & $8.23 \mathrm{e}-01$ & $7.99 \mathrm{e}-02$ & $9.65 \mathrm{e}-01$ & $3.09 \mathrm{e}-01$ \\
\cline { 2 - 6 } & ITAE & $5.26 \mathrm{e}+00$ & $5.02 \mathrm{e}-01$ & $5.91 \mathrm{e}+00$ & $2.56 \mathrm{e}+00$ \\
\hline \multirow{3}{*}{ PFLCPSS } & ISE & $1.75 \mathrm{e}-05$ & $2.03 \mathrm{e}-07$ & $2.05 \mathrm{e}-05$ & $7.15 \mathrm{e}-07$ \\
\cline { 2 - 6 } & IAE & $1.92 \mathrm{e}-02$ & $1.63 \mathrm{e}-04$ & $2.85 \mathrm{e}-02$ & $5.77 \mathrm{e}-05$ \\
\cline { 2 - 6 } & ITAE & $5.27 \mathrm{e}-02$ & $8.13 \mathrm{e}-04$ & $1.12 \mathrm{e}-01$ & $2.77 \mathrm{e}-03$ \\
\hline \multirow{3}{*}{ FLCPSS } & ISE & $1.75 \mathrm{e}-05$ & $2.95 \mathrm{e}-07$ & $2.05 \mathrm{e}-05$ & $6.17 \mathrm{e}-07$ \\
\cline { 2 - 6 } & IAE & $1.99 \mathrm{e}-02$ & $3.69 \mathrm{e}-04$ & $2.86 \mathrm{e}-02$ & $3.51 \mathrm{e}-04$ \\
\cline { 2 - 6 } & ITAE & $5.73 \mathrm{e}-02$ & $9.39 \mathrm{e}-04$ & $1.12 \mathrm{e}-01$ & $2.98 \mathrm{e}-03$ \\
\hline \multirow{3}{*}{ IT2FLCPSS } & ISE & $1.74 \mathrm{e}-05$ & $9.42 \mathrm{e}-08$ & $1.98 \mathrm{e}-05$ & $6.55 \mathrm{e}-08$ \\
\cline { 2 - 6 } & IAE & $1.92 \mathrm{e}-02$ & $1.63 \mathrm{e}-04$ & $2.85 \mathrm{e}-02$ & $3.51 \mathrm{e}-04$ \\
\cline { 2 - 6 } & ITAE & $4.92 \mathrm{e}-02$ & $8.35 \mathrm{e}-04$ & $1.11 \mathrm{e}-01$ & $3.13 \mathrm{e}-03$ \\
\hline
\end{tabular}

In Table $\mathrm{V}$, the behavior of controllers with the influence of model uncertainty is presented. Furthermore each value represents the average and the standard deviation of the three performance criteria which are calculated for 10 samples. A closer inspection of Table $\mathrm{V}$ reveals that the criteria values for T1 FLCPSS and IT2FLCPSS type-2 FLC are similar. This is due to the mitigation effect of these uncertainties by the FLS. In such a case, it is advisable to select a T1 FLCPSS since it is easier to implement. However, with the performance criteria values shown in Table IV one can suggest that at the lower values of ISE, IAE, and ITAE, the best system response is obtained when using an IT2FLCPSS. These results demonstrate the ability of the proposed controller to cope with an uncertain process and its potentiality to outperform its $\mathrm{T} 1$ counterpart.

TABLE V. PERFORMANCE CRITERIA VALUES FOR PSS-VARIOUS MODELS (SCENARIOS)

\begin{tabular}{|c|c|c|c|c|c|}
\hline \multirow{2}{*}{} & \multicolumn{4}{|c|}{$\begin{array}{c}\text { Scenarios \# } \\
\text { (Modeling uncertainty) }\end{array}$} \\
\cline { 3 - 6 } \multicolumn{2}{c|}{} & $\mathbf{1}$ & $\mathbf{2}$ & $\mathbf{3}$ & $\mathbf{4}$ \\
\hline \multirow{4}{*}{ ISE } & CPSS & 0.0197 & 0.0298 & 0.0735 & 0.0040 \\
\cline { 2 - 6 } & PFLCPSS & 0.0117 & 0.0196 & 0.0462 & 0.0007 \\
\cline { 2 - 6 } & FLCPSS & 0.0116 & 0.0200 & 0.0461 & 0.0005 \\
\cline { 2 - 6 } & IT2FLCPSS & 0.0118 & 0.0198 & 0.0471 & 0.0011 \\
\hline \multirow{4}{*}{ IAE } & CPSS & 4.6459 & 4.2780 & 6.2612 & 2.0789 \\
\cline { 2 - 6 } & PFLCPSS & 1.9978 & 2.6958 & 3.7928 & 1.1379 \\
\cline { 2 - 6 } & FLCPSS & 1.9626 & 2.6276 & 3.6820 & 0.9862 \\
\cline { 2 - 6 } & IT2FLCPSS & 2.1615 & 2.9320 & 4.2134 & 1.5209 \\
\hline \multirow{4}{*}{ ITAE } & CPSS & 6.7013 & 5.2684 & 8.0880 & 3.3461 \\
\cline { 2 - 6 } & PFLCPSS & 2.0698 & 3.0261 & 4.2960 & 1.6868 \\
\cline { 2 - 6 } & FLCPSS & 2.0020 & 2.8350 & 4.0587 & 1.3968 \\
\cline { 2 - 6 } & IT2FLCPSS & 2.5119 & 3.7904 & 5.3436 & 2.7344 \\
\hline
\end{tabular}

\section{CONCLUSION}

The application of three fuzzy logic-based control algorithms as power system stabilizers is described in this paper. Time domain simulation and modal analysis performed with various stabilizers, over a wide range of operating conditions with different disturbances, have demonstrated the effectiveness and robustness of this control algorithm. A comparison of the performance of the four algorithms (the three fuzzy logic-based and the conventional) shows that the three controllers generally provide better performance than a fixed parameter conventional stabilizer. Although all four algorithms provide acceptable performance, IT2FLCPSS is the most suitable when the presence of measurement uncertainties (noise) is more pronounced. Also, due to its simplicity it can be easily implemented for the control of large scale power systems.

\section{REFERENCES}

[1] T. Hiyama, "Development of fuzzy logic power system stabilizer and further studies", 1999 IEEE International Conference on Systems, Man, and Cybernetics, Tokyo, Japan, October 12-15, 1999

[2] M. Sanaye-Pasand, O. P. Malik, "Implementation and laboratory test results for a new fuzzy logic based PSS", 1996 Canadian Conference on Electrical and Computer Engineering, Calgary, Canada, May, 26-29, 1996

[3] M. A. M. Hassan, O. P. Malik, "Implementation and laboratory test results for a fuzzy logic based self-tuned power system stabilizer", IEEE Transactions on Energy Conversion, Vol. 8, No. 2, pp. 221-228, 1993

[4] Q. Liang, J. M. Mendel, "An introduction to type-2 TSK fuzzy logic systems", 1999 IEEE International Fuzzy Systems, Seoul, South Korea, August, 22-25, 1999

[5] S. Barkat, A. Tlemcani, H. Nouri, "Noninteracting adaptive control of pmsm using interval type-2 fuzzy logic systems", IEEE Transactions on Fuzzy Systems, Vol. 19, No. 5, pp. 925-936, 2011 
[6] X. Lu, M. Liu, J. Liu, "Design and optimization of interval type-2 fuzzy logic controller for delta parallel robot trajectory control", International Journal of Fuzzy Systems, Vol. 17, No. 1, pp. 595-608, 2015

[7] T. Kumbasar, H. Hagras, "A self-tuning zslices-based general type-2 fuzzy picontroller", IEEE Transactions on Fuzzy Systems, Vol. 23, No. 1, pp. 991-1013, 2015

[8] A. Padhi, Hardware Realization of Interval Type 2 Fuzzy Logic Controller, PhD Thesis, National Institute of Technology, Rourkela, India, 2015

[9] K. Su, S. Huang, C. Yang, "Development of robotic grasping gripper based on smart fuzzy controller", International Journal of Fuzzy Systems, Vol. 19, No. 1, pp. 190-206, 2017

[10] E. Nechadi, M. Harmas, A. Hamzaoui, N. Essounbouli, "Type-2 fuzzy based adaptive synergetic power system control", Electric Power Systems Research, Vol. 88, pp. 9-15, 2012

[11] Z. Sun, N. Wang, D. Srinivasan, Y. Bi, "Optimal tunning of type-2 fuzzy logic power system stabilizer based on differential evolution algorithm", International Journal of Electrical Power and Energy Systems, Vol. 62, pp. $19-28,2014$

[12] S. Kamel, B. Ziyad, H. M. Naguib, A. Mouloud, R. Mohamed, "An indirect adaptive type-2 fuzzy sliding mode PSS design to damp power system oscillations", 7th International Conference on Modelling, Identification and Control, Sousse, Tunisia, December 18-20, 2015

[13] A. Abbadi, L. Nezli, D. Boukhetala, "A nonlinear voltage controller based on interval type 2 fuzzy logic control system for multimachine power systems", International Journal of Electrical Power and Energy Systems, Vol. 45, No. 1, pp. 456-467, 2013

[14] M. Tripathy, S. Mishra, "Interval type-2-based thyristor controlled series capacitor to improve power system stability", IET Generation, Transmission \& Distribution, Vol. 5, No. 2, pp. 209-222, 2011

[15] N. Karnik, J. Mendel, Q. Liang, "Type-2 fuzzy logic systems", IEEE Transactions on Fuzzy Systems, Vol. 7, No. 6, pp. 643-658, 1999

[16] K. Sebaa, M. Boudour, "Optimal locations and tuning of robust power system stabilizer using genetic algorithms", Electric Power Systems Research, Vol. 79, No. 2, pp. 406-416, 2009

[17] G. Rogers, Power System Oscillations, Springer, 2000

[18] P. Kundur, Power System Stability and Control, EPRI, 1994

[19] K. Sebaa, H. Gueguen, M. Boudour, "Mixed integer non-linear programming via the cross-entropy approach for power system stabilisers location and tuning", IET Generation, Transmission \& Distribution, Vol. 4, No. 8, pp. 928-939, 2010 